\title{
Image enhancement on the INVIS integrated night vision surveillance and observation system
}

\author{
Judith Dijk, Klamer Schutte, Alexander Toet, Maarten Hogervorst ${ }^{\S}$ \\ * TNO Defence, Security and Safety, P.O. Box 96864, 2509 JG, The Hague, The Netherlands \\ email: Judith.Dijk@tno.nl \\ ${ }^{\S}$ TNO Defence, Security and Safety, P.O. Box 23, 3769 ZG, Soesterberg, The Netherlands
}

\begin{abstract}
We present the design and first field trial results of the INVIS integrated night vision surveillance and observation system, in particular for the image enhancement techniques implemented. The INVIS is an all-day-andnight all-weather navigation and surveillance tool, combining three-band cameras. We present a processing pipeline for this system. The image quality of all individual sensor signals is enhanced through Dynamic Noise Reduction and Dynamic Super Resolution. The quality of the thermal image can be enhanced through SceneBased Non-Uniformity Correction (SBNUC). The images are fused using natural tone mapping techniques. The contrast in the image can be improved using Local Adaptive Contrast Enhancement, applied before or after the tone mapping. These results show that the image enhancement techniques have an added value for image fusion systems.
\end{abstract}

Keywords: image fusion, image enhancement, false color, natural color, real-time fusion, thermal infrared, intensified imagery.

\section{INTRODUCTION}

For all military operations, situational awareness is of great importance. This situational awareness can be obtained using cameras. The current trend is that more and more operations are shifted from daytime to night. This increases the need for night-time imagery. In night time operations, image-intensified cameras are used next to thermal infrared cameras. The advantage of image-intensified cameras with respect to infrared cameras is that they better allow for visual identification. Infrared cameras, however, can be used for detection of hot objects. A system that contains both an image intensified and an infrared camera can combine the benefits of both.

In recent years TNO developed the the TRICLOBS ${ }^{1}$ TRI-band Color Low-light OBServation system in which three cameras are combined. The sensor suite of the system comprises two digital image intensifiers (sensitive in the visual and near-infrared) and a thermal (LWIR) camera. The cameras are combined so that the optical axis of the imagery is equal. The combination of the two intensified images, representing the visual and near-infrared bands, can be used to display the resulting multiband nightvision image in realistic daytime colors, using the Color-the-Night color mapping principle. ${ }^{2,3}$ This mapping can also be deployed to enhance the visibility of thermal targets that are camouflaged in the visual and near-infrared bands,

The all-day all-weather INVIS Integrated Night Vision surveillance and observation System ${ }^{4}$ integrates the TRICLOBS $^{1}$ TRI-band Color Low-light OBServation system with a synthetic 3D scene generation system, advanced image fusion and image processing capabilities. Position information is provided by a 3D digital GPS system. The viewing direction of the system (the azimuth and tilt of its optical axis) is provided by a digital compass.

The image quality of both the camera output and the fused images are enhanced using image enhancement algorithms. The quality of the single images is enhanced through Dynamic Superresolution (DSR2) and temporal noise reduction (DSR1). The quality of the thermal image can be further enhanced through SceneBased Non-Uniformity Correction (SBNUC). The fused image is enhanced using Local Adaptive Contrast Enhancement (LACE).

Enhanced and Synthetic Vision 2010, edited by Jeff J. Güell, Kenneth L. Bernier, Proc. of SPIE Vol. 7689, 768909 - ( 2010 SPIE · CCC code: 0277-786X/10/\$18 - doi: 10.1117/12.850785 


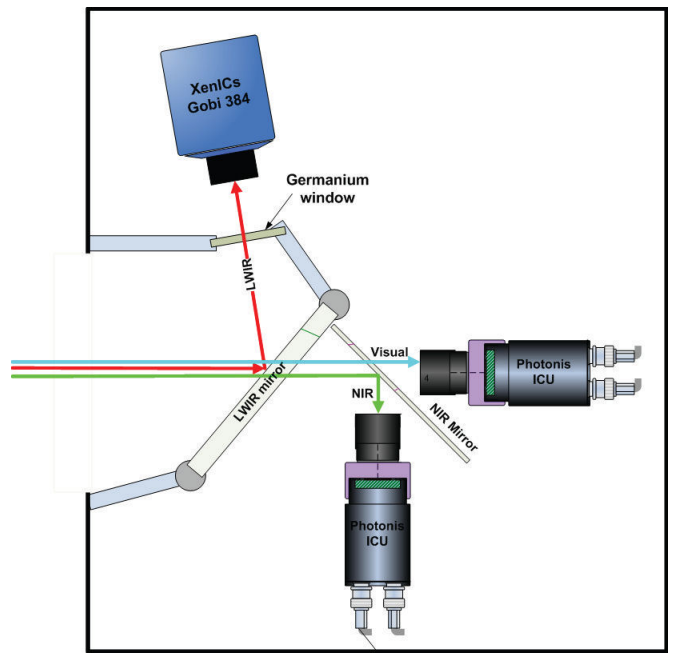

(a) layout of the system

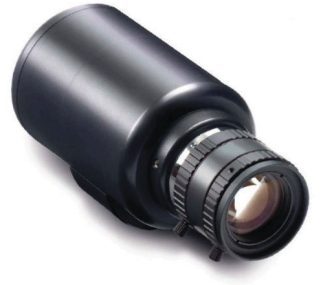

(b) Image intensifier $\left(I^{2}\right)$ : ICU (Photonis)

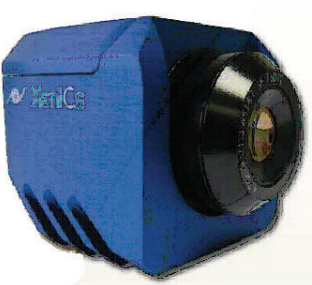

(c) thermal camera (TIR): Gobi (XenICs)

Figure 1. Overview of the INVIS system. In (a) an schematic overview of the cameras and the optical paths is shown. In (b) and (c) the cameras are shown.

In this paper, we present the performance of these image enhancement techniques for the different individual sensors and the fused image. The INVIS system is described in more detail in section 2 . The image enhancement techniques that applied and the processing pipeline that we propose are described in section 3 . The results of the experiments are presented in section 4 . The results are discussed in section 5.

\section{INVIS SYSTEM}

The INVIS integrates the TRICLOBS ${ }^{1}$ TRI-band Color Low-light OBServation system with advanced image fusion and processing capabilities. The INVIS system comprises a three-band nightvision sensor suite, GPS and inertial sensors, a geometric 3D scene model, and a laptop to dynamically process (i.e. fuse, colorize, and enhance) the imagery and to generate synthetic images from the 3D scene model. In this paper we do not describe the synthetic image generation system that is also part of the INVIS system. The system, its cameras and their optical paths are shown in figure $1 \mathrm{a}$. The system is described in more detail by Toet et.al. ${ }^{4}$ The sensor suite and color mapping procedure are described in the rest of this section. The image enhancement algorithms follow in the next section.

\subsection{Sensor Suite}

The TRICLOBS contains three cameras: two image intensifiers and one thermal camera. The two image intensifiers $\left(I^{2}\right)$ are high resolution (1280x960) Photonis PP3000U Intensified Camera Units (ICUs, see figure 1(b): www.photonis.com). The ICU is a low light level, intensified CMOS camera. Both ICU's are equipped with Pentax C2514M CCTV lenses. The signal is read through the PAL or NTSC composite video signal output (ITU-R BT.656-4, 640x480 pixels). The camera signal is filtered so that the camera is sensitive in the visual and near infrared spectrum, respectively.

The thermal camera (TIR) is a XenICs Gobi 384 uncooled a-Si infrared microbolometer (figure 1(c): www.xenics.com). It has a $384 \times 288$ pixel focal plane array, and a spectral sensitivity range of $8-14 \mu \mathrm{m}$, which is the range of most interest for outdoor applications. It is equipped with an Ophir supIR18mm F/1 lens (www.ophiropt.com). 


\subsection{Color mapping}

The INVIS system software comprises a color remapping procedure and several image enhancement procedures. The image enhancment algorithms are described in more detail in the next section. Color remapping serves to represent the fused sensor signals in natural daytime colors, which makes nighttime imagery easier to interpret (more intuitive). ${ }^{5}$ It can also be used to selectively enhance target conspicuity by increasing target color contrast. The signals from the three nighttime cameras are fused and represented in natural daytime colors using the Color-the-Night false color remapping procedure. ${ }^{2,3}$ The object colors only depend on the multiband sensor values, and are independent of the image content. As a result, objects keep the same color over time when recorded with a moving camera.

\section{IMAGE ENHANCEMENT TECHNIQUES}

Image enhancement can be applied to an image or a sequence of images. The purpose of these techniques is to improve the quality of the images presented to an observer. In this paper we evaluate the benefits of four image enhancement techniques: temporal noise reduction (DSR1), super-resolution reconstruction (DSR2), scene based non uniformity correction (SBNUC) and local adaptive contrast enhancement (LACE). All four algorithms can be implemented on hardware, as well in a real-time software application. The latency of the combined algorithms is smaller than three frames. In previous experiments ${ }^{6,7}$ we evaluated the effect of these algorithms for $I^{2}$ cameras in a test environment. Here, we apply the algorithms on real outdoor data.

\subsection{Noise reduction and super-resolution reconstruction}

In case multiple frames of a scene are available, temporal filtering can produce better results. When the camera and scene is stationary or the frames can be aligned accurately, temporal filtering will not deteriorate the scene structure such as for instance spatial filtering does. Temporal noise reduction can be done using averaging of the aligned frames, or using more complex techniques such as super resolution. With these techniques the spatial resolution of the images can also be improved. An overview of super-resolution techniques is given by Park. ${ }^{8}$ Schutte et al. ${ }^{9}$ presented the Dynamic Super Resolution (DSR) algorithm, which is implemented in real-time op a PC platform. Next to super-resolution (DSR2) the DSR algorithm can also be used for noise reduction (DSR1). In this case the parameters of the algorithm are set such that the number of the pixels is not increased. For super-resolution simultaneously the number of pixels is increased and the noise is reduced. As the imagery of the image intensifier is noise limited, the main added value is expected for noise reduction. The infrared camera is resolution limited, so here resolution enhancement is expected to provide more benefit. The detectors in the thermal camera have different response characteristics. This introduces another type of noise: non-uniformities. Scene-Based Non-Uniformity Correction (SBNUC) serves to correct for the non-uniformities. SBNUC is based on the assumption that the scene content moves slowly relative to the frame rate, whereas the non-uniformities are semi-static. Using accurate motion estimation, the statistics per pixel can be used to correct the non-uniformity for this particular pixel.

\subsection{Local contrast enhancement}

Next to noise reduction, images can also be enhanced using contrast enhancement. The goal is to visualise more details in the image. To enhance the contrast in an image several methods are described in literature. The most simple way to enhance the contrast is by adjusting the image to the available range, so-called global contrast stretching. This will help in cases were only part of the available range is used, but will fail in situations were the range used in one part of the image differs much from other parts. To enhance the contrast in a part of the available contrast range gamma manipulation can be done. In this case details in part of the lightness range of the image will be enhanced, at the cost of decreasing the lightness in other parts of the image. Another global method is histogram equalization, were the lightness values are changed so that the histogram of the image are as flat as possible. The main disadvantage of this method is that the output images are not so natural anymore, and that dmall objects often are saturated.

To enhance local contrasts, local adaptive contrast enhancement can be used. The idea of local contrast enhancement is that the processing depends on features in a local region. Narenda and Fitch ${ }^{10}$ propose a method in which the local statistics are described by its local mean and variance. Using a local region means 


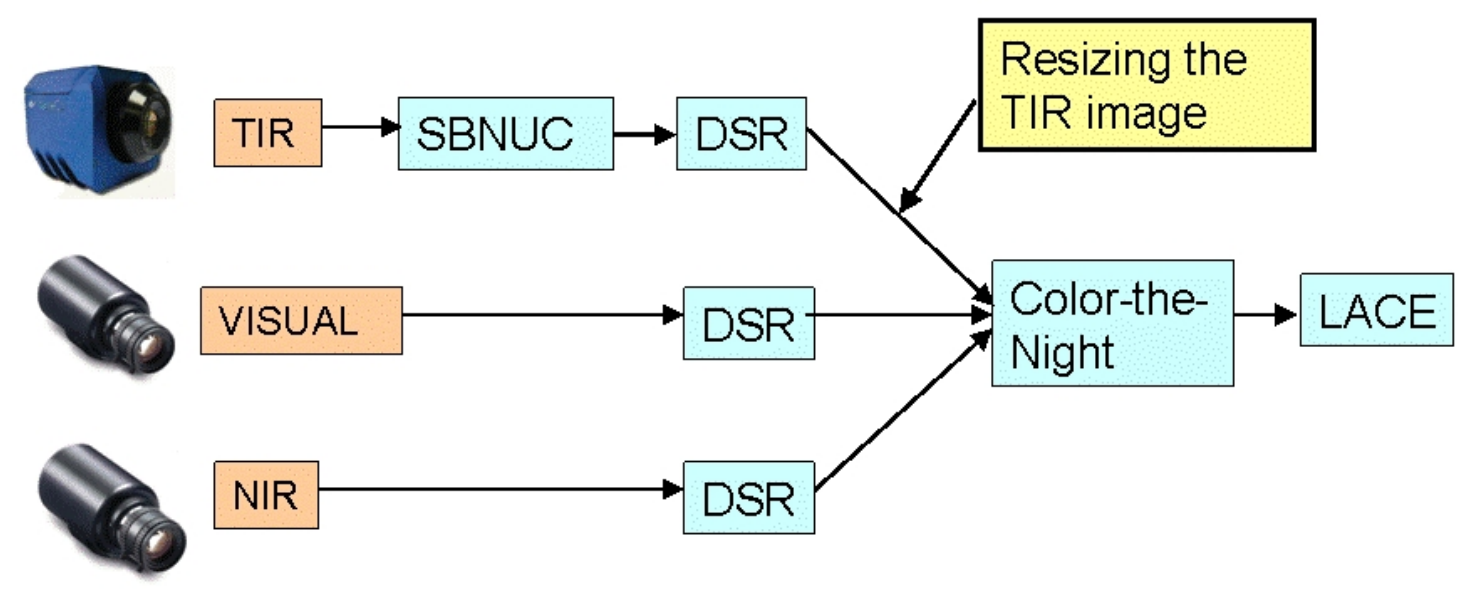

Figure 2. Overview of processing pipeline for the INVIS system.

that the contrast is enhanced in a specific scale in the image, for instance only small details. To avoid having to chose a specific scale, the contrast can be adjusted at several scales in the image.

In our evaluation we use $\mathrm{LACE}^{11}$, a grey-value algorithm which is based on the method of Narenda and Finch, but extended to a number of scales. As this algorithm drastically changes the intensity, this image enhancement will normally be applied after fusing the inputs of the different cameras. This algorithm can be used to obtain images showing all interesting features in an image. We apply two different settings: low LACE and high LACE. The setting for low LACE is such that the contrast is enhanced without producing much artifacts. For the high LACE setting details are enhanced more, at the cost of more artefacts. Dijk et al. ${ }^{12}$ proposed to perform the local contrast enhancement on color images by performing the contrast enhancement on the luminance channel, after which we recombine it with its colors.

\subsection{Processing pipeline}

In Figure 2 we present the pipeline of algorithms proposed. DSR and SBNUC do not change the dynamic range of the pixels and will be performed on the separate images. After this the color mapping will be applied. LACE is applied on the combined image, as this method drastically changes the dynamic range. The TIR image needs to be interpolated to the same size after the DSR algorithm, otherwise resolution enhancement will not have an effect.

\section{RESULTS}

\subsection{Experimental setup}

We tested the INVIS system during a nocturnal data collection trial in the field. The location was in Marnehuizen, a Dutch MOUT village (Google Earth: Latitude 53.386293 and Longitude 6.262944). The ambient light levels were below 0.01 lux.

\subsection{Image processing results}

In Figure 3 and 4 results are given of noise reduction. On the $I^{2}$ images it is very clear that a significant improvement of the Signal to Noise Ratio (SNR) is obtained using the temporal noise reduction as provided by the DSR1 algorithm. On the TIR image significant improvement of the SNR is observed using fixed pattern noise reduction provided by SBNUC and temporal noise reduction provided by DSR1.

In Figure 5 results are given of resolution enhancement, with enlarged region of interests in Figure 6 . It can be seen that the DSR results on the $I^{2}$ image are blurry, this is the result of the low input SNR. In effect 


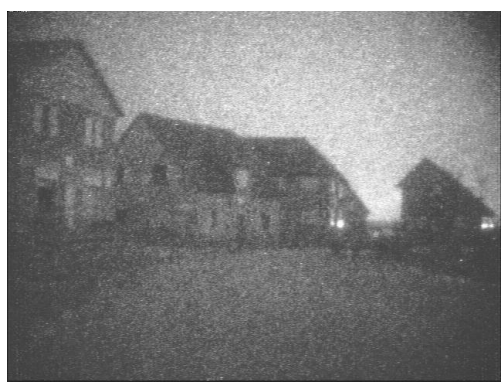

NIR original

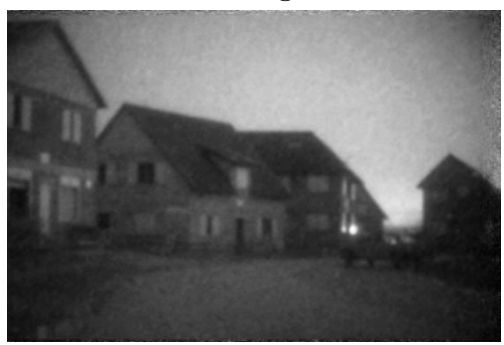

NIR DSR1

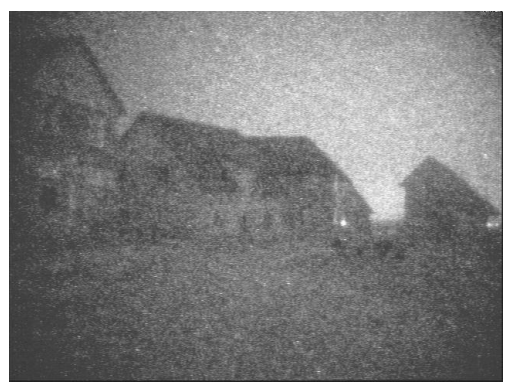

VISUAL original

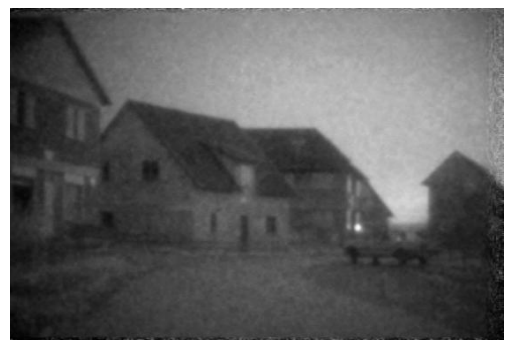

VISUAL DSR1

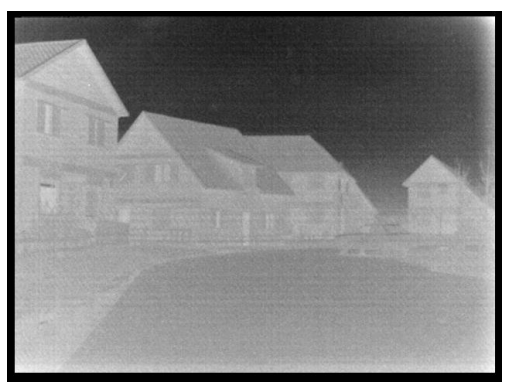

TIR original

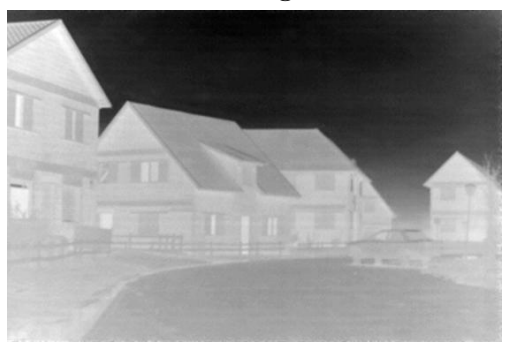

TIR DSR1 + SBNUC

Figure 3. Temporal noise reduction using the DSR1 algoritm and SBNUC.

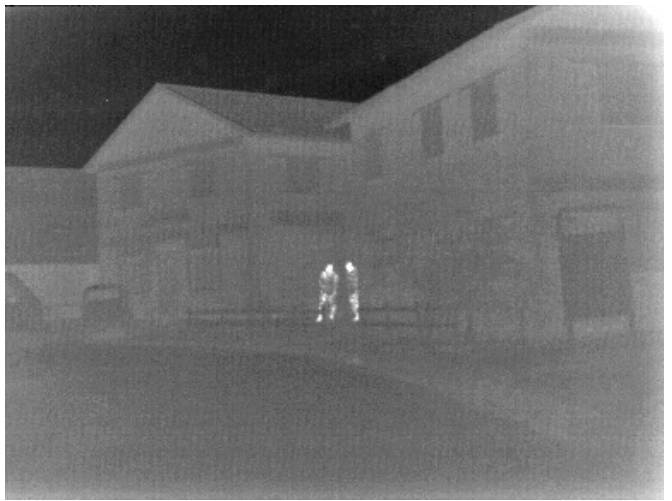

original

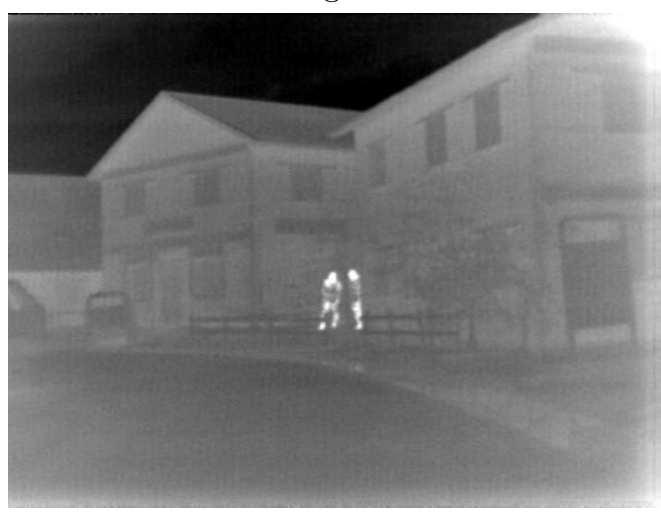

DSR1

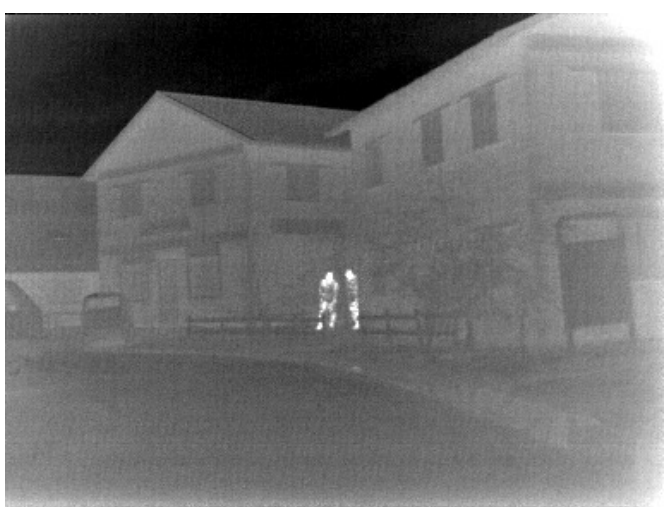

SBNUC

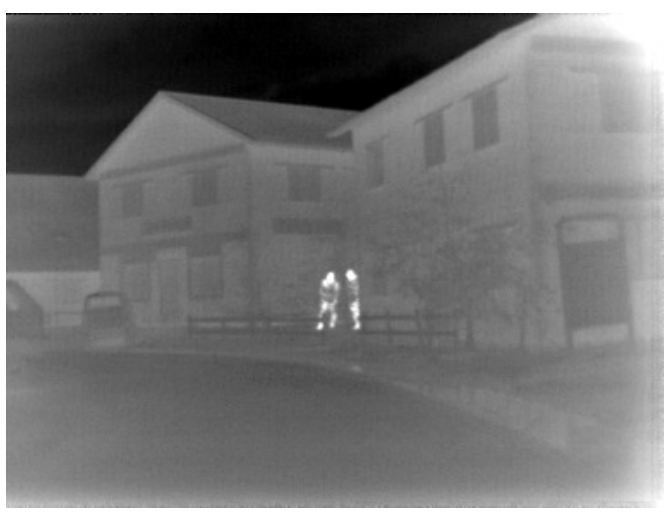

SBNUC + DSR1

Figure 4. Temporal noise reduction using the DSR1 algoritm and SBNUC on the TIR imagery. 


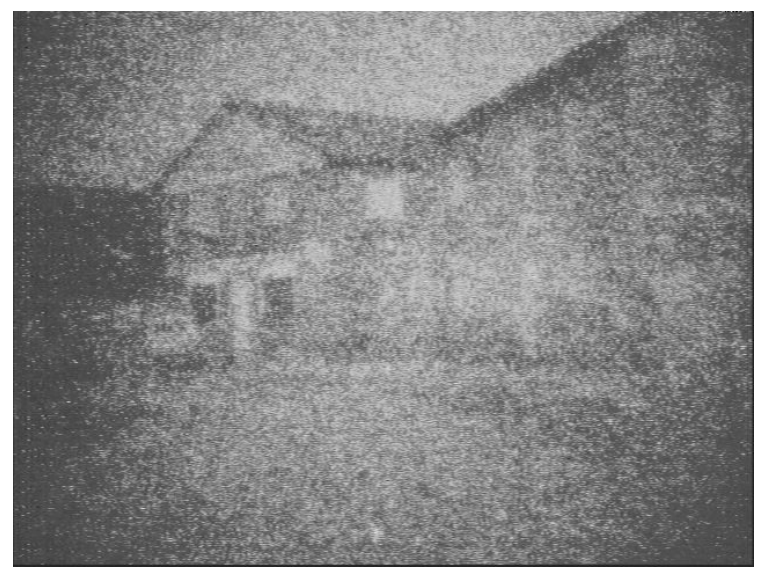

NIR original

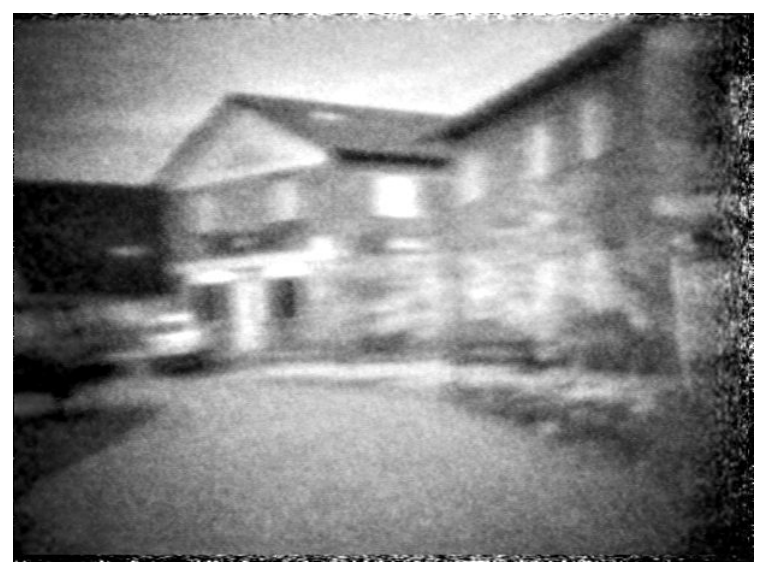

NIR DSR1

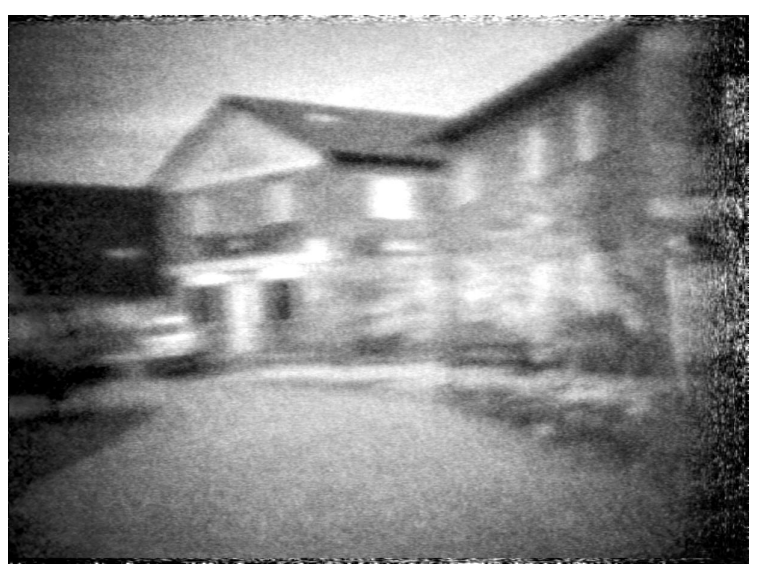

NIR DSR2

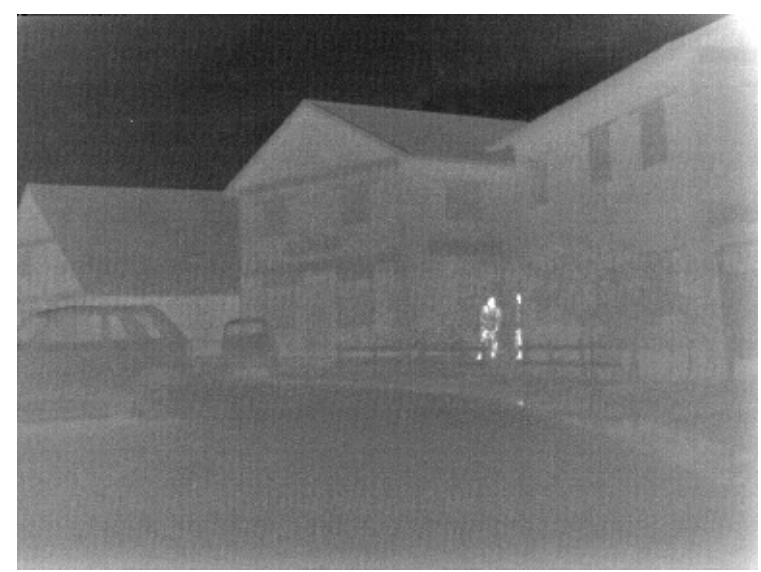

TIR original

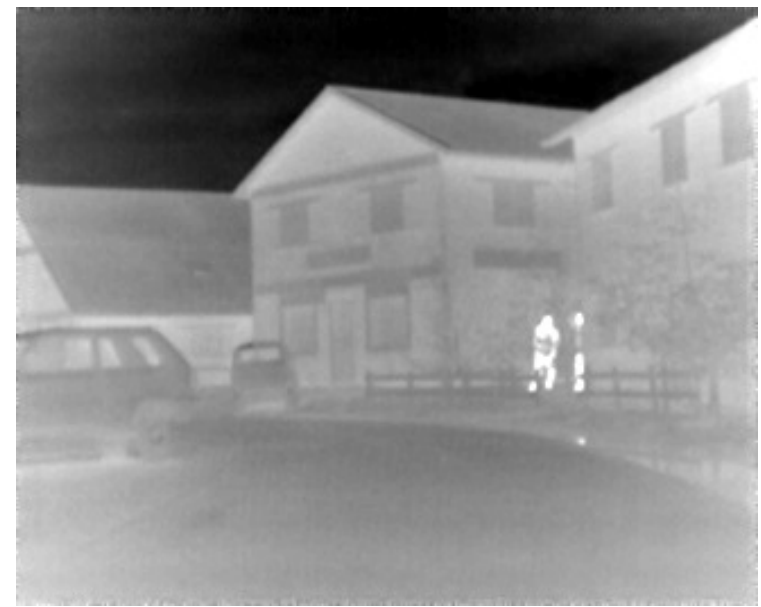

TIR DSR1 + SBNUC

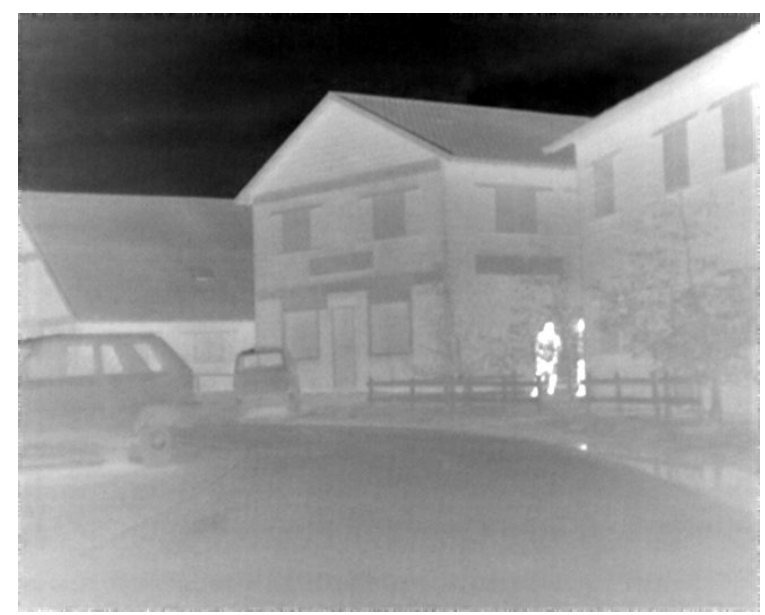

TIR DSR2 + SBNUC

Figure 5. Comparison of noise reduction (DSR1) and resolution enhancement (DSR2). 
this translates to no improved image quality after DSR2 application. The white, somewhat blurred, objects in front of the right house are a number of trees. These appear almost white in NIR imagery.

The SNR on the TIR images, especially after SBNUC, is much better. This results in a better motion estimation, which in turn translates to a improved image quality using DSR2 compared to DSR1.

Figure 7 provides results with the LACE contrast enhancement algorithm on the separate imagery. The second row shows for both the $I^{2}$ and the TIR images that applying LACE without noise reduction increases both noise and the image contrast. With proper noise reduction applied, as in the fourth row, LACE does provide better insight into image details. In the last row is observed that applying more LACE does result in even better visualisation of low contrast details, at the expense of more pronounced residual noise and more artificial images.

Figure 8 provides results with the LACE contrast enhancement algorithm in combination with the Colorthe-Night algorithm. The second row shows that the Color-the-Night algorithm can be applied on the processed images. In the third row low LACE is applied, both before and after the color mapping. It can be seen that applying LACE after the color mapping gives a better preservation of the colors of the original images. In the fourth row high LACE is applied, also before and after the color mapping. It can be seen that the contrast enhancement on the right image (LACE after $\mathrm{CtN}$ ) is somewhat too high, resulting in more perceived noise and less color. The amount of LACE on the left image is less pronounced. This indicates that the CtN algorithm reduces the effect of LACE on the separate images.

In Figure 9 and Figure 10 the impact of moving objects to DSR results is visualized. The DSR algorithm is equipped with moving object robustness features, limiting the impact of moving object artefacts. It can be observed that the small moving objects, i.e. the persons in Figure 9, are well visible in the enhanced imagery. On the other hand, in Figure 10 significant deblurring is observed all over the field. This is caused by a failure in the motion estimation process within the DSR algorithm due to too much motion in the image.

\section{CONCLUSIONS AND DISCUSSION}

In this paper results are given of image enhancement techniques on imagery of the INVIS integrated night vision surveillance and observation system. We presented a processing pipeline for the different algorithms. Noise reduction and resolution enhancement are applied before the color tone mapping, local adaptice contrast enhancement after this mapping. All techniques applied on the single sensor do not change the range of the sensor. This provides for a better natural tone mapping.

Both the $I^{2}$ and the TIR images greatly benefit from noise reduction. For the $I^{2}$ this noise reduction is obtained by the DSR1 (motion corrected averaging) algorithm, while for the TIR a combination of SBNUC (Scene Based Non-Uniformity Correction) and DSR1 is used.

For the TIR imagery an additional enhancement is obtained by using the DSR2 instead of the DSR1 algorithm, i.e. adding super resolution reconstruction capability. This compensates for the relative low input resolution of the uncooled IR imager used. On the $I^{2}$ imagery no added value was seen when using super resolution reconstruction. This suggests that the input data is more noise limited than resolution limited.

The DSR algorithm proved to be robust to smaller moving objects, like persons walking through the scene. However, with extreme scene motion patterns, as seen with evolving smoke clouds, the DSR algorithm seemed unable to perform adequately.

In addition the imagery has been enhanced using LACE. It clearly showed that for effective contrast enhancement initial noise reduction as obtained by DSR1 and SBNUC really benefits. In imagery with LACE low contrast details are greatly improved. Amount of contrast enhancement is shown to be dependent of actual system application.

The contrast in the image is improved using Local Adaptive Contrast Enhancement. This contrast enhancement can be applied before and after the tone mapping. It is shown that applying the contrast enhancement before the tone mapping changes the colors of the fused image. Applying contrast enhancement after the tone mapping results in fused images which still have the daytime color appearance as the original fused image. Another advantage is that the LACE algorithm has to be applied only once. Resizing the TIR image to the 


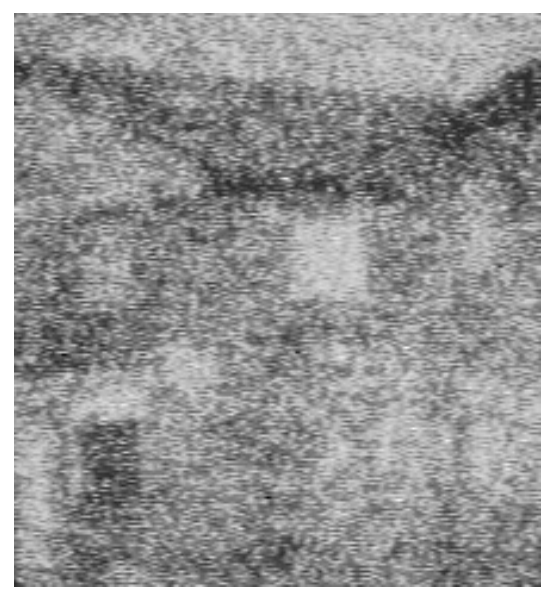

NIR original

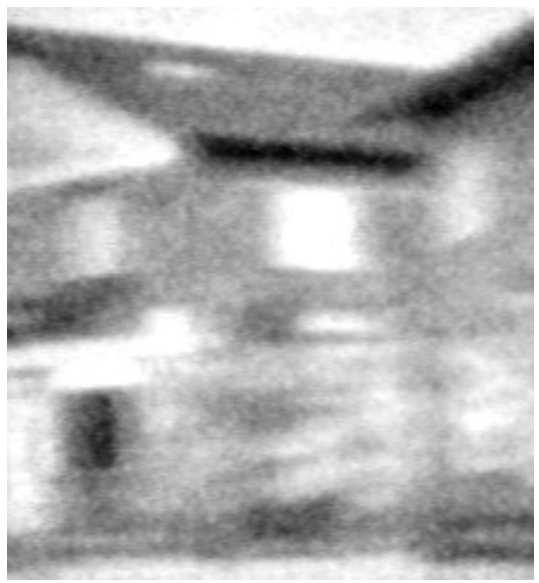

NIR DSR1

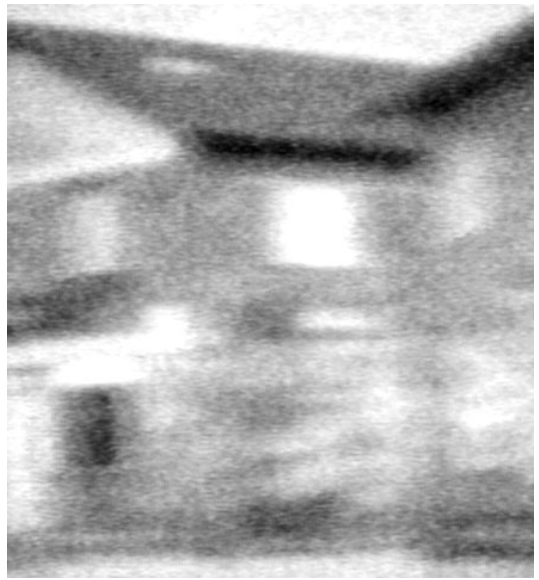

NIR DSR2

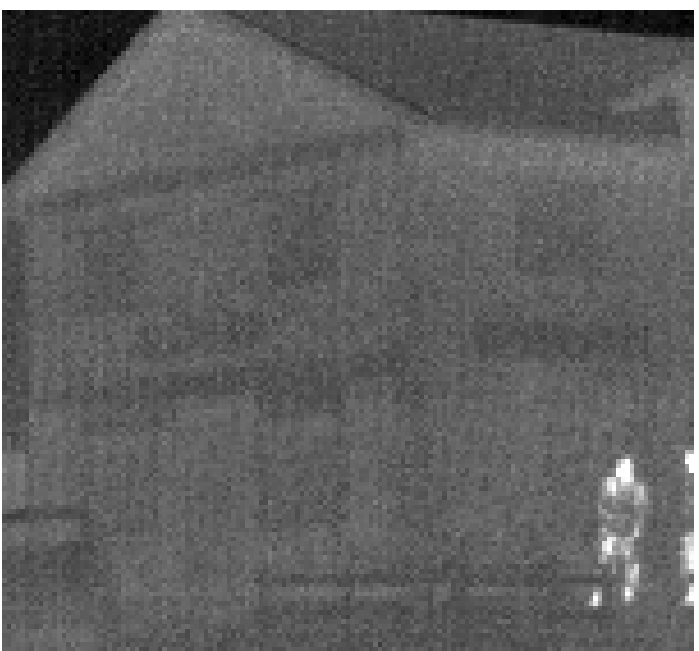

TIR original

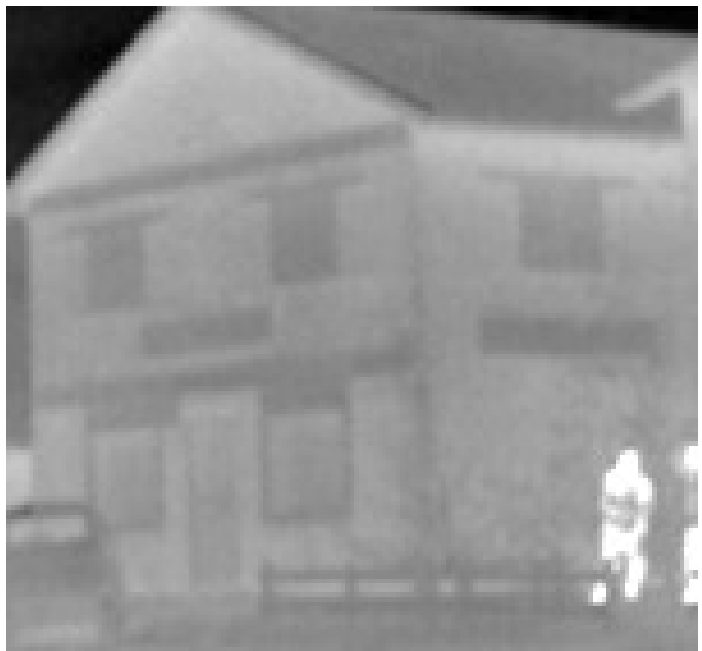

TIR DSR1 + SBNUC

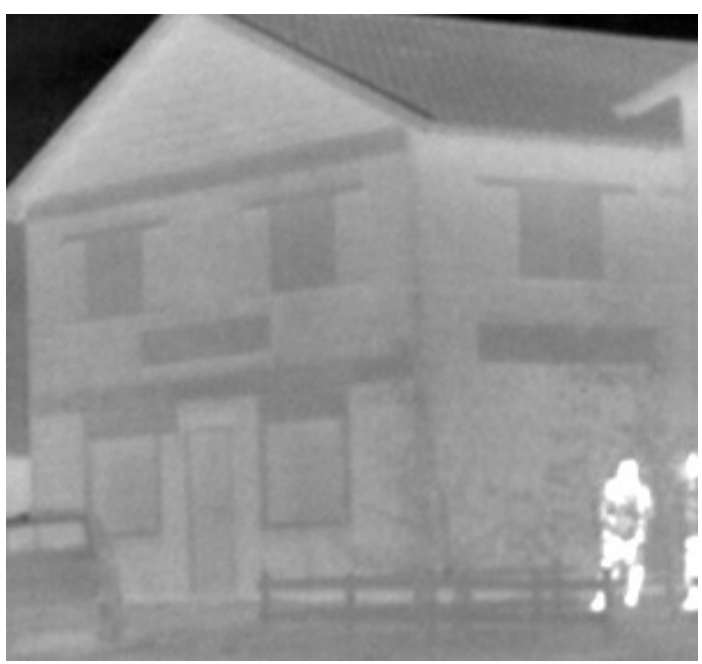

TIR DSR2 + SBNUC

Figure 6. Enlarged regions of interest of DSR1 and DSR2 results. 


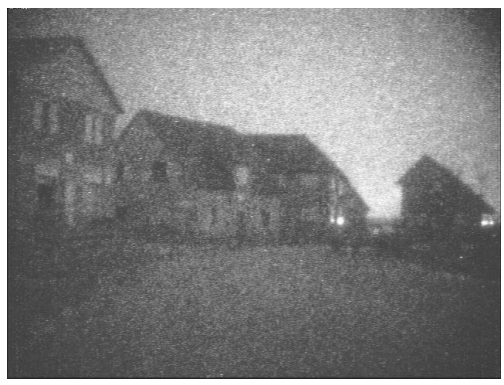

original NIR

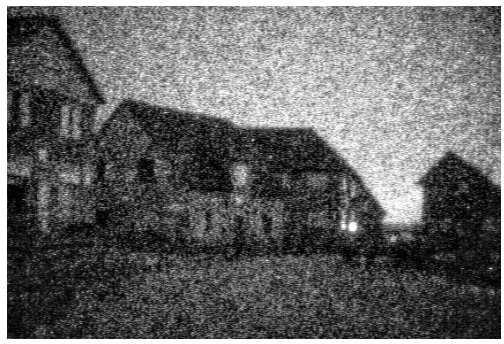

low LACE

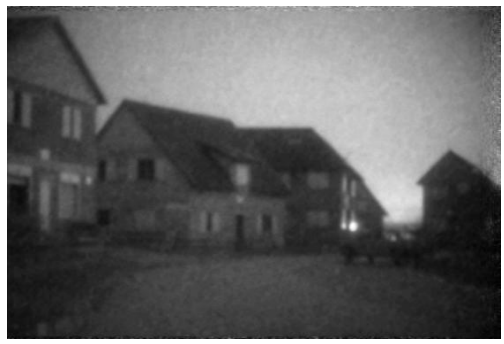

DSR1

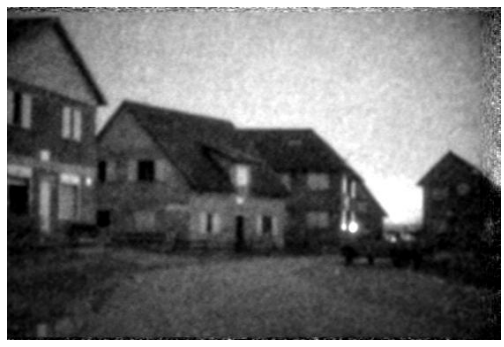

DSR1 + low LACE

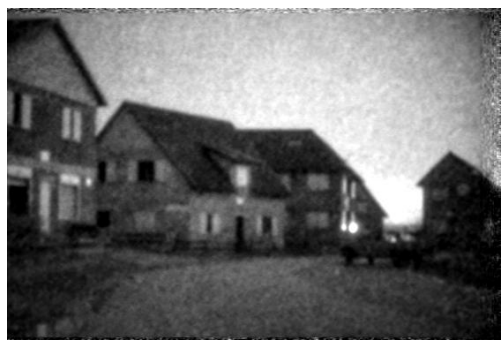

DSR1 + high LACE

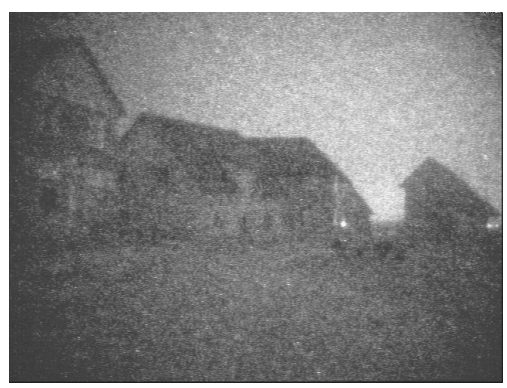

original VISUAL

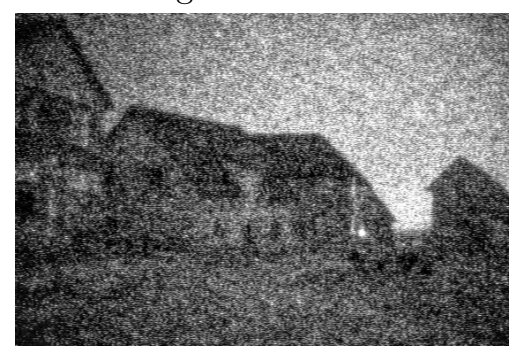

low LACE

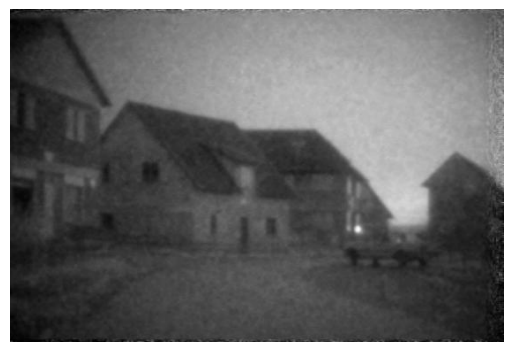

DSR1

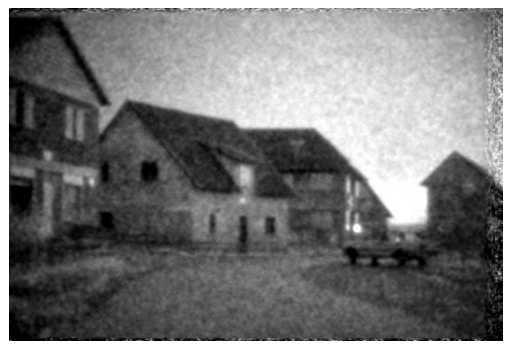

DSR1 + low LACE

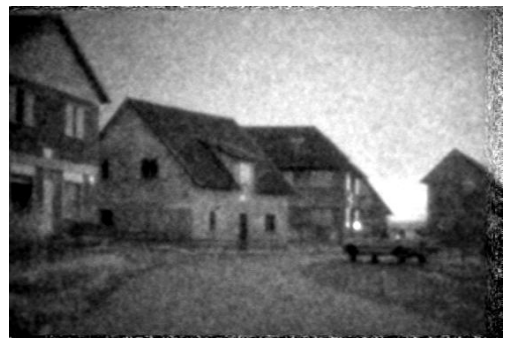

DSR1 + high LACE

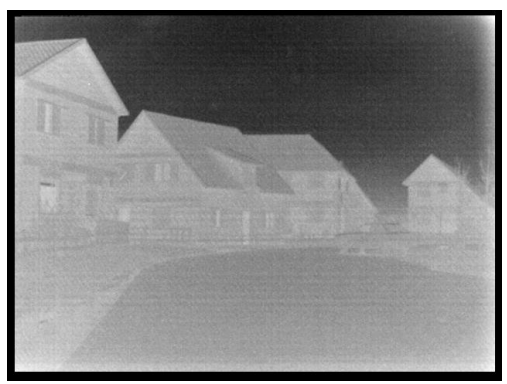

original IR

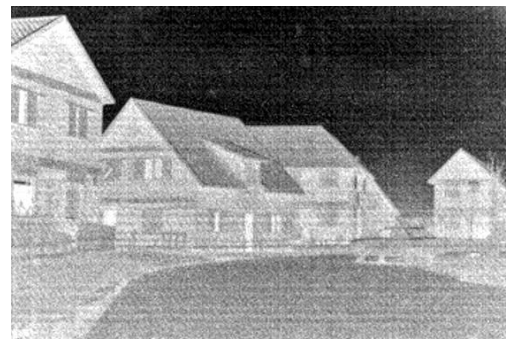

low LACE

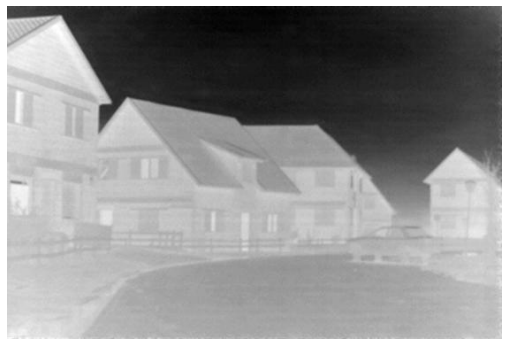

DSR1

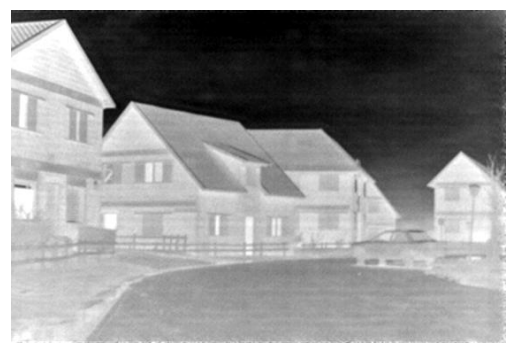

DSR1 + low LACE

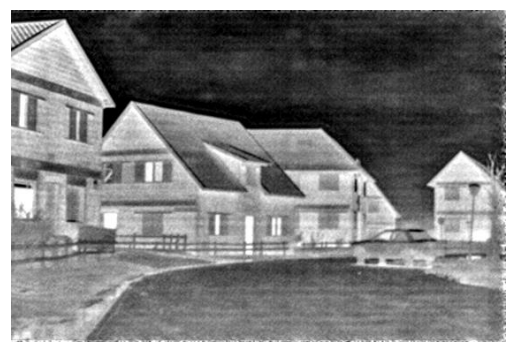

DSR1 + high LACE

Figure 7. Results for LACE with and without combination with DSR1 on separate images 


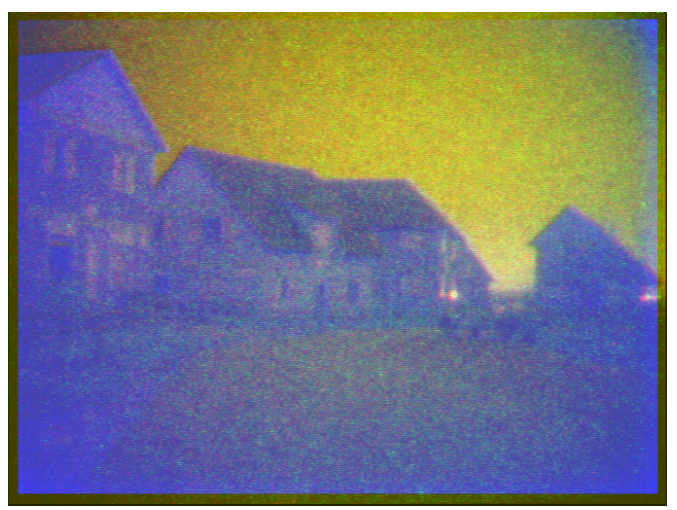

RGB

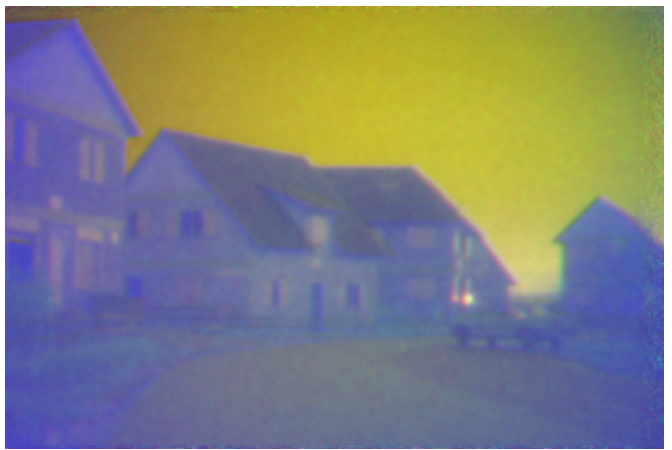

$\mathrm{RGB}+\mathrm{CtN}$

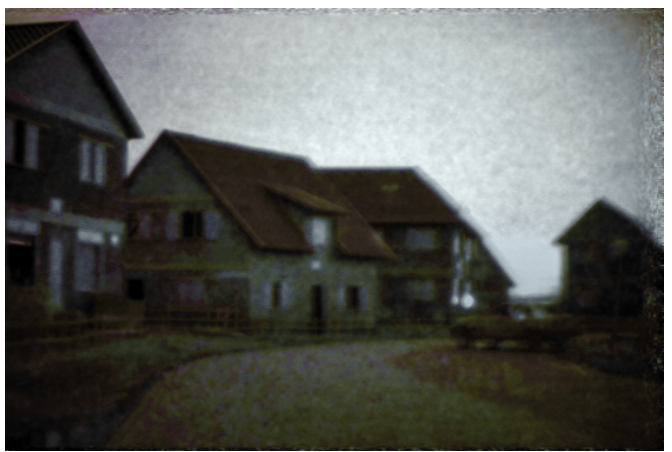

DSR1, low LACE before CtN

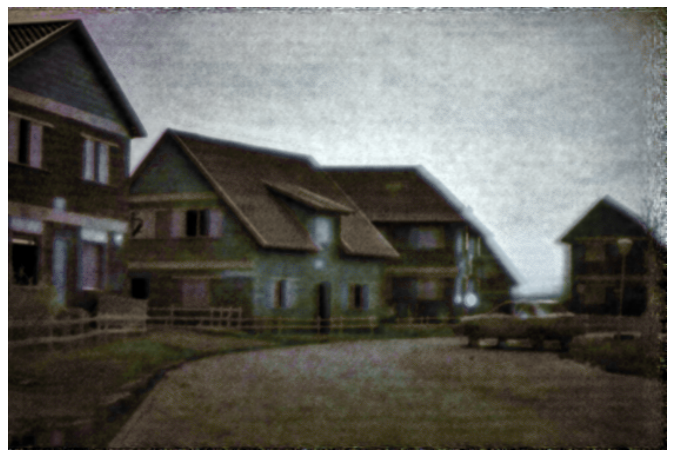

DSR1, high LACE before CtN

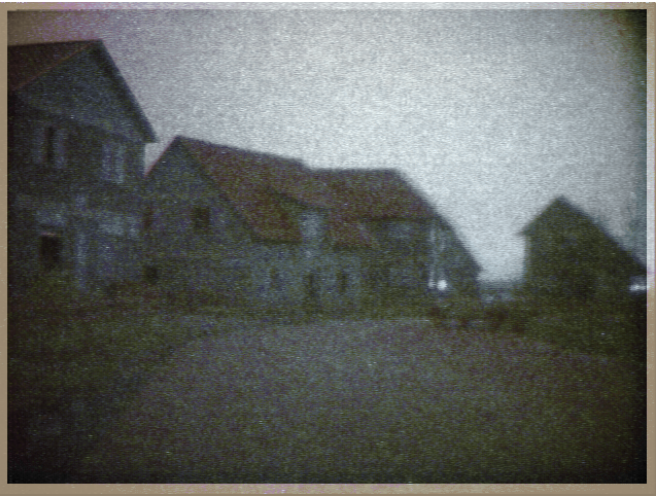

Color-the-Night

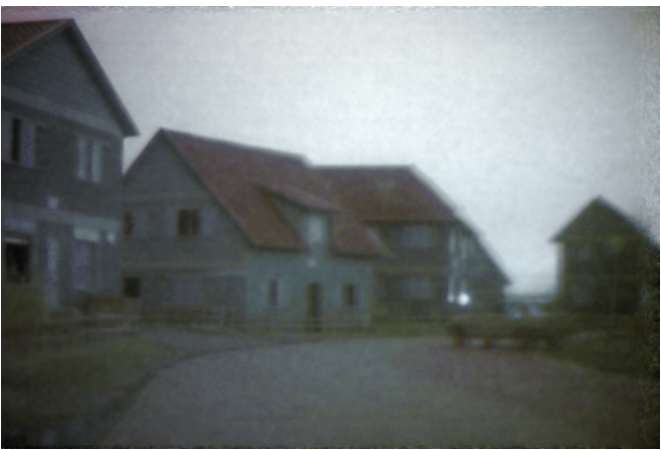

$\mathrm{DSR} 1+\mathrm{CtN}$

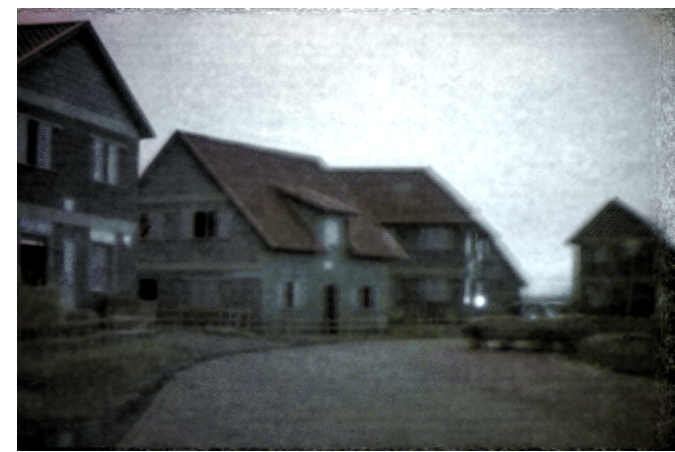

DSR1, low LACE after CtN

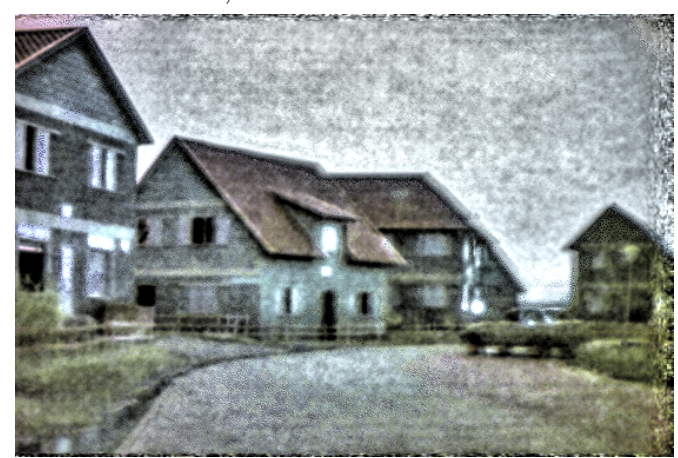

DSR1, high LACE after CtN

Figure 8. Results for Color-the-Night in combination with DSR and LACE 


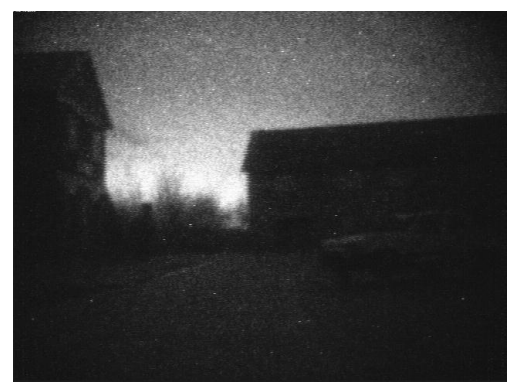

original NIR

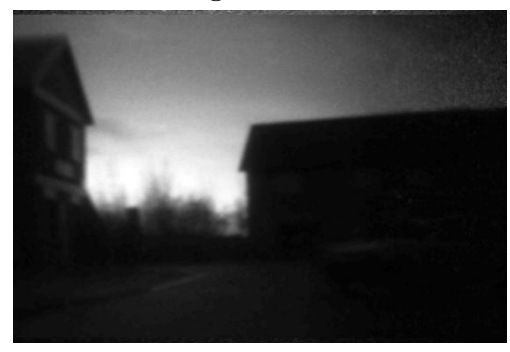

DSR1+low LACE NIR

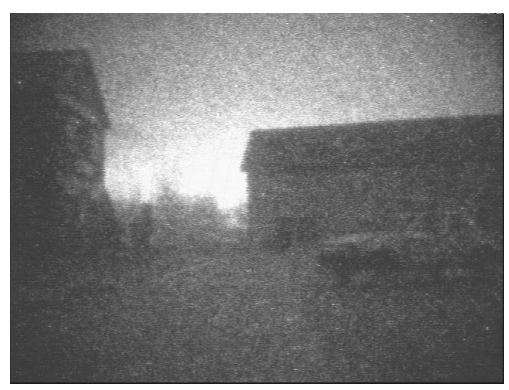

original VISUAL

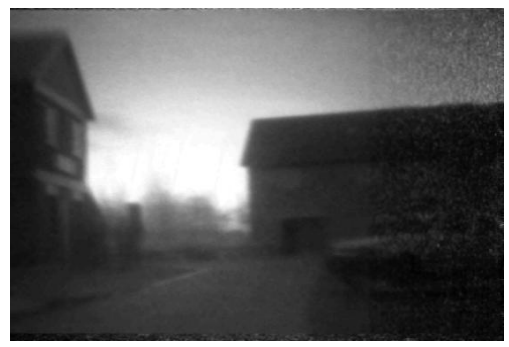

DSR1 + low LACE VISUAL

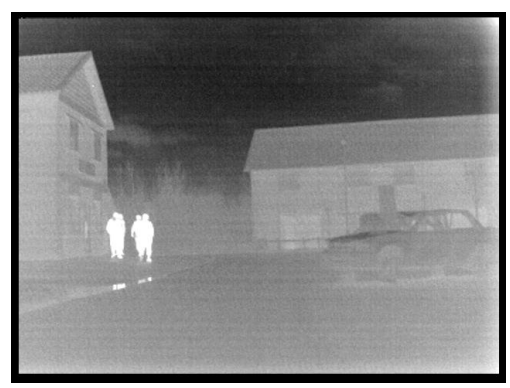

original IR

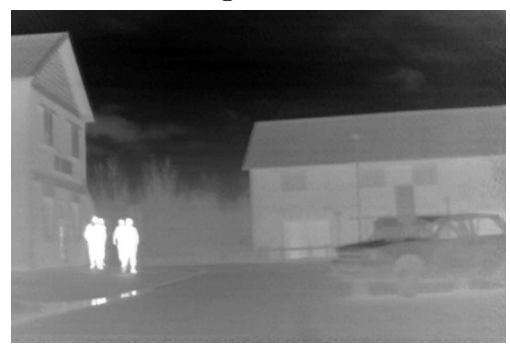

DSR1+SBNUC+low LACE IR

Figure 9. Results for images including moving objects.

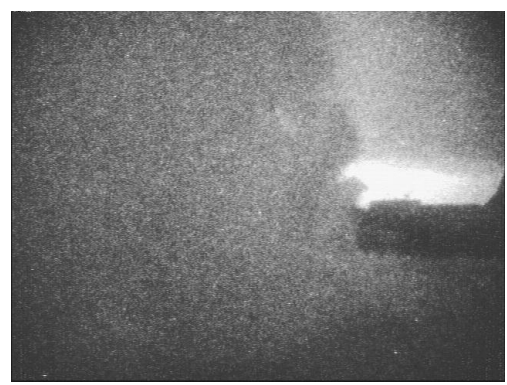

original NIR

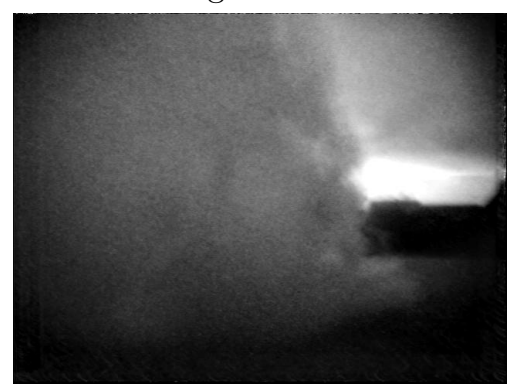

DSR1+low LACE NIR

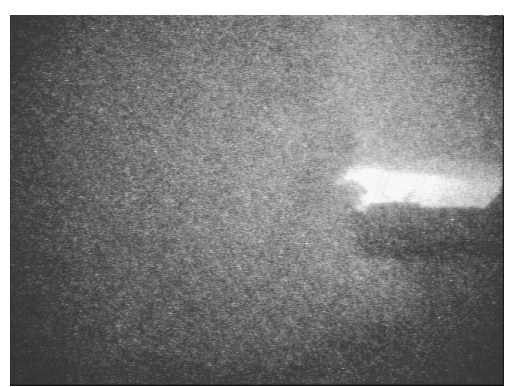

original VISUAL

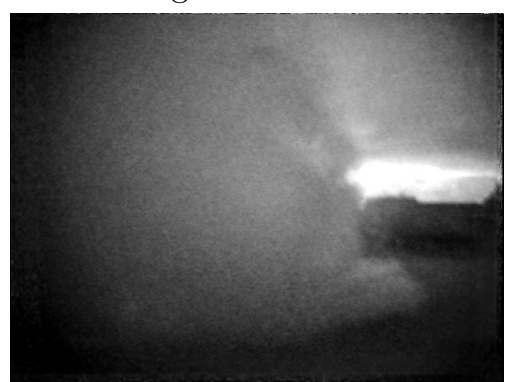

DSR1 + low LACE VISUAL

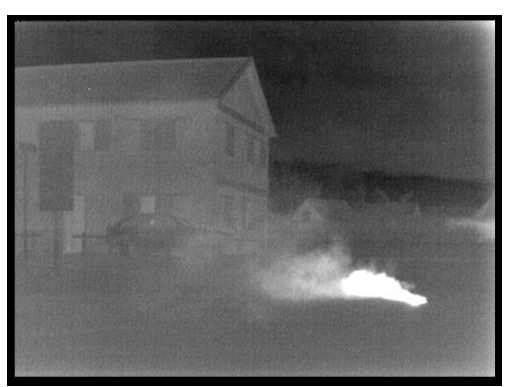

original IR

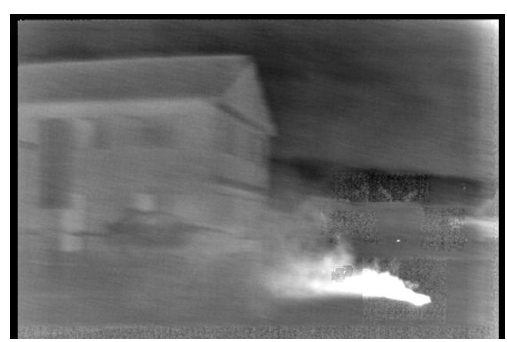

DSR1+SBNUC+low LACE IR

Figure 10. Results for images with smoke in the scene

same size of the $I^{2}$ images has to be done just before the color mapping, otherwise the resolution enhancement cannot be performed properly.

Overall, the results in this paper show that the image enhancement techniques have an added value for this type of image fusion systems. The quality of the separate and fused images improves significantly using these algorithms. 


\section{Acknowledgement}

This effort was partly sponsored by the Air Force Office of Scientific Research, Air Force Material Command, USAF, under grant number FA8655-09-3095. The U.S. Government is authorized to reproduce and distribute reprints for Governmental purpose notwithstanding any copyright notation thereon.

\section{REFERENCES}

[1] Alexander Toet and Maarten. A. Hogervorst, "TRICLOBS portable triband color lowlight observation system," in Proc. SPIE, 2009, vol. 7345.

[2] Alexander Toet, "Color the night: applying daytime colors to nighttime imagery," Enhanced and Synthetic Vision, pp. 168-178, 2003.

[3] Alexander Toet, "Natural colour mapping for multiband nighttime imagery," Information Fusion, vol. 4, no. 3, pp. 155-166, 2003.

[4] Alexander Toet, Maarten A. Hogervorst, Judith Dijk, and Rob van Son, "Invis integrated night vision surveillance and observation system," in Proc. SPIE, 2010, vol. Proc. SPIE vol. 7689.

[5] Maarten A. Hogervorst and Alexander Toet, "Evaluation of a color fused dual-band nvg-sensor," in Proc. SPIE, 2009, vol. 7345.

[6] Judith Dijk, Piet Bijl, and Henri Bouma, "Performance evaluation of image enhancement techniques on a digital image-intensifier," Proc of SPIE, vol. 7300, 2009.

[7] Judith Dijk, Piet Bijl, and Adam W. M. van Eekeren", "Performance evaluation of image enhancement techniques on night vision imagery," Proc of SPIE, vol. 7662, 2010.

[8] S.C. Park, M.K. Park, and M.G. Kang, "Super-resolution image reconstruction: a technical overview," IEEE Signal Processing Magazine, vol. 20, no. 3, pp. 21-36, 2003.

[9] Klamer Schutte, Dirk-Jan J. de Lange, and Sebastiaan P. van den Broek, "Signal conditioning algorithms for enhanced tactical sensor imagery," in Proc. SPIE: Infrared Imaging Systems: Design, Analysis, Modeling, and Testing XIV, 2003, vol. 5076, pp. 92-100.

[10] P. M. Narenda and R.C. Finch, "Real-time adaptive contrast enhancement," IEEE transactions on pattern analysis and machine intelligence, vol. 3, no. 6, pp. 655-661, 1981.

[11] Klamer Schutte, "Multi-scale adaptive gain control of ir images," in Proc. SPIE, 1997, vol. 3061, pp. 906-914.

[12] Judith Dijk, Richard J.M. den Hollander, John G.M. Schavemaker, and Klamer Schutte, "Local adaptive contrast enhancement for color images," Proc of SPIE, vol. 6575, 2007. 